\title{
LA INEQUIDAD EN SALUD. SU DESARROLLO HISTÓRICO
}

\author{
Oswaldo Salaverry García ${ }^{1,2, a}$
}

\section{RESUMEN}

La inequidad en salud, centro de los debates contemporáneos sobre salud pública, se asienta en bases filosóficas e históricas que se remontan a la idea de justicia de la Grecia clásica. Se revisa la aproximación Aristotélica sobre la justicia distributiva y su forma superior la epiekeia o equidad y como esta evoluciona durante el medioevo y la modernidad hasta llegar a ser el centro del debate para pensadores tan diversos como el liberal Rawls y el premio nobel Amartya Sen. Sobre ese debate conceptual se resume la versión de la Organización Mundial de la Salud que vincula equidad con determinantes de la salud e intenta operativizarla a través de la provisión equitativa de servicios de salud.

Palabras clave: Desigualdades en la Salud; Salud Pública; Historia de la medicina (fuente: DeCS BIREME).

\section{INEQUITY IN HEALTH. IT'SHISTORICAL DEVELOPMENT}

\section{ABSTRACT}

Health inequity, main issue of contemporary debates on public health, is based on philosophical and historical concepts that date back to the idea of justice from classic Greece. The Aristotelian approach on distributive justice and its higher form, epiekeia or equity, has been reviewed, as well as how this evolves from the Middle Ages and modernity to the heart of the debate of a variety of thinkers such as liberal Rawls and Nobel laureate Amartya Sen. On this conceptual debate lies the World Health Organization version that links equity to health determinants and intends to make it operational through the equitable provision of health services.

Key words: Health inequalities; Public Health; History of medicine (source: MeSH NLM).

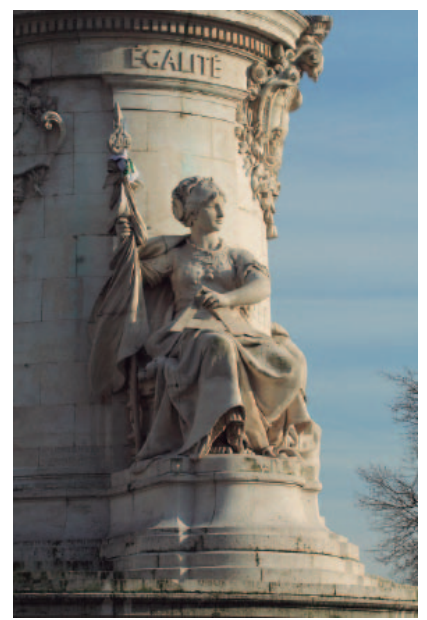

Figura 1. Allégorie a l'égalité

Monumento central (parte inferior izquierda de la estatua) de la Place de la Republiqué, Paris (Francia).

La igualdad, l'égalité, es uno de los tres principios de la revolución francesa, bajo el cual se creía que "el saber que todos somos iguales" eliminaría las barreras, discrepancias y luchas por conceptos como edad, sexo, opinión, origen, religión y capacidades de cada persona.

\footnotetext{
Centro Nacional de Salud Intercultural, Instituto Nacional de Salud. Lima, Perú.

Facultad de Medicina, Universidad Nacional Mayor de San Marcos. Lima, Perú.

Médico, doctor en medicina

Recibido: 18/11/2013 Aprobado: 20-11-13
}

Citar como: Salaverry García O. La inequidad en salud. Su desarrollo histórico. Rev Peru Med Exp Salud Publica. 2013;30(4):709-13. 
El discurso político-sanitario contemporáneo, bien sea de los organismos multilaterales de salud, y los integrantes del sistema de las Naciones Unidas, está estrechamente vinculado con la idea de equidad, o en su forma más concreta con la búsqueda de la equidad en salud. Las políticas nacionales en salud de los países, en particular los latinoamericanos reflejan ese mismo objetivo. Pareciera haberse alcanzado un consenso universal en este punto; la equidad es el desideratum de la salud pública.

Esta aparente unanimidad es frágil pues tanto los planes y programas de los diferentes sistemas nacionales de salud, como los planes impulsados y promovidos por los organismos multilaterales difieren notablemente en la operativización de las metas con que buscan alcanzar la equidad, reflejando así una limitación del aparente consenso. Sin duda eso se debe a la estrecha vinculación del concepto de equidad con uno muy cercano, el de igualdad, del cual se ha generado y diferenciado a través de un complejo proceso fundamentalmente filosófico. El caso de la equidad en salud, tema de más reciente aparición, es necesario analizarlo desde una perspectiva histórica, para poder comprender como se ha percibido las relaciones entre igualdad y desigualdad y entre justicia y equidad cuando se refieren a la salud. Un análisis histórico-filosófico de su génesis y evolución nos acerca a entender la complejidad subyacente a un discurso que suele limitarse al enunciado de lo políticamente correcto.

\section{LA DESIGUALDAD EN EL ORIGEN}

La evidencia cotidiana nos muestra como seres físicamente diferentes; si apelamos a nuestras habilidades o competencias, las diferencias se acentúan en algunos casos y disminuyen en otros. Así fue percibida la naturaleza humana desde tiempos prehistóricos y sobre esa base se estructuraron las distintas civilizaciones de la antigüedad hasta el periodo moderno, considerando la desigualdad humana como la base "natural" de las relaciones interpersonales y también de la relación con el estado. La idea de una subyacente igualdad de todos los seres humanos surge tempranamente pero desde una perspectiva religiosa que la limita al ámbito moral; la "hermandad" de los integrantes de una comunidad religiosa no se extendía a su lugar en la sociedad o su jerarquía, por el contrario el factor religioso fue utilizado para justificar las prerrogativas de los reyes o lideres por su vinculación especial con la divinidad. Tal es la idea subyacente en la "divinidad" de los reyes, seres humanos que trascendían su condición por un mandato divino o por una filiación directa con lo divino.
En el tema de salud, la omnipresencia de la enfermedad y la muerte sin distinguir a nobles y gobernantes del común de las personas tenía una explicación religiosa igualadora que refería una etapa primigenia en la cual la enfermedad y la muerte no existían introduciéndose en el mundo por una falta o pecado, afectando desde entonces a todos por igual; no existiría acción terrenal que pudiera revertir esa igualdad. Esto se plasma en el Coro de Antígona describiendo al hombre: Nada habrá en el futuro / A lo que él sin recursos se encamine / Tan sólo el medio de evitar la muerte / Nunca se habrá de encontrar: / Más para dolencias de imposible cura / Modos de escape tiene ya ingeniados ${ }^{(1)}$.

El sentido de "justicia" no estaba sin embargo alejado de esta interpretación de la salud pues si bien la enfermedad sería un componente "natural" de la vida, consecuencia de la voluntad divina, las buenas obras o el cumplimiento de las disposiciones religiosas conllevaría a la salud. Un ejemplo es la plegaria de Apiladad, un personaje babilónico, que ante la enfermedad que lo aqueja le recuerda al Dios que ha cumplido con los preceptos religiosos y por tanto le corresponde estar sano y el dios "debe" brindarle nuevamente salud (2). Otro ejemplo es la historia de Job en el antiguo testamento, cuestionado por sus propios compañeros para que identifique la falta o pecado cometido para hacerse acreedor a la enfermedad que lo aqueja, pero él se limita a reiterar su absoluto cumplimiento de los deberes religiosos y por tanto se resigna con aceptar decisiones divinas inexplicables para él.

Debemos esperar al surgimiento de la filosofía griega para encontrar las primeras discusiones sobre la equidad como una forma superior de la justicia. Si bien el tema fue tratado por los presocráticos toma consistencia en Platón pero especialmente en Aristóteles que en su formulación ética y especialmente en la "Ética a Nicómaco" orienta el debate sobre la justicia que hasta hoy marca la discusión filosófica.

\section{EQUIDAD EN LA CULTURA CLÁSICA}

El termino igualdad proviene, en las lenguas romances, del término latino aequitas, (francés "equité", italiano "equitá", rumano "echitate", catalán "equitat"). La aequitas latina se entiende como la traducción del

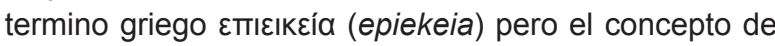
aequitas latino difiere y no tiene la sutileza del concepto griego. Epieikeia como se translitera en alfabeto latino, es un concepto desarrollado inicialmente por Platón y luego por Aristóteles y que se forma a partir del prefijo $\varepsilon \pi$, que significa "sobre, encima, luego, después o también" y de la raíz દıkoo (eikos) que se traduce como 
"razonable, justo, conveniente o esperable". Para ambos filósofos la Epieikeia es una forma de justicia y por tanto de aplicación de las Leyes, pero difieren notablemente en el sentido que tiene. Platón en "La Republica", una de sus obras tempranas, considera que la sabiduría práctica del juez sabio y prudente es superior a las leyes y por tanto debe buscarse a este juez para que la aplique; posteriormente se da cuenta que es difícil o imposible encontrar el hombre sabio y por tanto en "Las leyes", una obra tardía, promueve la aplicación de Leyes generales que sustituyan al sabio. La epiekeia o equidad se convierte así para Platón como una forma débil de la justicia, una que se aleja de lo ideal y se tiñe de misericordia.

Para Aristóteles la epiekeia no es una excepción a la ley, es una forma superior de ella, pues si bien la ley es justa la epiekeia o equidad es más justa aun pues incluye los casos concretos, algo que la simple ley general no puede hacer. Si las personas no son iguales, no tendrán una igualdad en la manera como serán tratadas. De aquí vienen las disputas y las contiendas cuando las personas, sobre un pie de igualdad, no obtienen partes iguales, o cuando personas, en pie de desigualdad, tienen y obtienen un tratamiento igual. Lo que es equitativo, aun siendo justo, no lo es de conformidad con la ley; es como un mejoramiento de lo que es justo según la ley ${ }^{(3)}$.

La interpretación aristotélica de la equidad fue la base del derecho romano como se verifica en Ulpiano, jurista romano del siglo II citado en el Digesto: luris praecepta haec sunt: honeste vivere, alterum non laedere, suum ciuque tribuere ius (Los mandatos del derecho son estos: vivir honradamente, no molestar a los demás, dar a cada cual lo suyo). Claro está que el "dar a cada cual lo suyo" es interpretado en la antigüedad desde la perspectiva de las profundas diferencias "naturales" existentes en los hombres y que entre otras cosas permitía la esclavitud.

Durante la edad media Santo Tomás de Aquino (1225-1274) el máximo representante del escolasticismo reinterpreta a Aristóteles derivando el termino latino aequus del griego

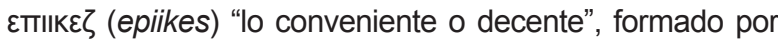

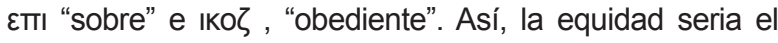
sometimiento a la ley por encima de lo conveniente, un retorno a la interpretación absoluta de la Ley.

\section{DESIGUALDAD E INEQUIDAD EN EL MUNDO MODERNO}

El desarrollo científico y tecnológico que dio lugar al surgimiento del mundo moderno generó nuevas formas de desigualdad al tiempo que también originó un creciente debate sobre la igualdad. Por una parte el proceso de urbanización dependiente de la industrialización hizo crecer grandes masas de trabajadores que vivían en terribles condiciones, la desigualdad se manifestó en falta de acceso a servicios básicos y en una creciente diferenciación en los niveles de ingreso, todo ello fue generando mayores disparidades en la salud. Las tensiones sociales sin embargo se dirigieron más hacia las condiciones políticas y un paulatino proceso de democratización atraviesa todo occidente, por un parte con hitos como la Revolución Francesa (Figura 1), la declaración de la independencia norteamericana, el proceso de la independencia en América del sur y revueltas como la de 1848 en Europa. Los temas de salud sin embargo y las inequidades tienen un prolongado letargo. Durante el siglo XIX a la desigualdad entre los individuos, aun ampliamente aceptada como "natural", se le añadió un componente racial perverso. En 1853 el filósofo francés Joseph Arthur de Gobineau publica "Essai sur le l'inegalite des races humanes" donde desarrolla una historia de las grandes civilizaciones atribuyendo su apogeo y desaparición a factores raciales. Esta obra, mezcla de vulgarizaciones científicas y prejuicios, influenció profundamente algunos círculos intelectuales europeos incluyendo a Richard Wagner quien sublimó el mito de la raza aria que, posteriormente, asumiría el nazismo. No faltaron respuestas a la propuesta de la jerarquización racial con la raza aria en la cúspide, siendo la principal la de un intelectual haitiano Joseph Anténor Firmin quien en 1885 publica "De l'égalité des races humaines. Anthropologie positive", rebatiendo los argumentos de Gobineau y señalando los grandes logros de las diversas etnias o como se denominaba entonces las razas.

\section{EL DEBATE CONTEMPORÁNEO}

La discusión sobre la equidad no puede prescindir de las opiniones contemporáneas del filósofo norteamericano John Rawls, quien en su muy difundido e influyente libro "A theory of justice" (4), originalmente publicado en 1971, plantea dos principios: el primero que todas las personas deben tener un derecho igual al conjunto más amplio de libertades básicas iguales que sea compatible con las libertades que disfrutan los demás; segundo, que las desigualdades sociales y económicas deben resolverse a su vez aplicando otros dos principios; el principio de la diferencia que consiste en que las acciones deben dirigirse de modo que resulten en el mayor beneficio posible para los integrantes menos aventajados de la sociedad y el principio de igualdad de oportunidades que establece que las oportunidades en la sociedad deben estar disponibles para todas las personas bajo similares 
condiciones. La influencia de Rawls considerado uno de los pensadores liberales más importantes del siglo $\mathrm{XX}$ y su perspectiva de la equidad es revisada particularmente por Amartya Sen, premio nobel de Economía 1988, pero desde una perspectiva completamente opuesta al liberalismo de Rawls pero que coincide con él en el análisis de la equidad como parte del debate de la justicia distributiva de la tradición aristotélica occidental. Sen desarrolla una teoría acerca de la inequidad en salud, que parte de establecer que cualquier teoría acerca de la justicia incorpora un concepto de igualdad, sea este en términos distributivos del ingreso o de derechos o cualquiera otro concebible, pero la justicia social se vincula principalmente con la salud por el carácter multidimensional de esta última. Hace referencia que pese a que contamos con el concepto más general de equidad es necesario desarrollar el de equidad en salud porque en sus palabras: las libertades y posibilidades que somos capaces de ejercer dependen de nuestros logros en salud. Porque no podemos hacer muchas cosas si estamos discapacitados o incesantemente abrumados por la enfermedad y son muy pocas las que podemos hacer si no estamos vivos ${ }^{(5)}$.

Frente a la frecuente confusión entre equidad en salud y distribución de la atención sanitaria, tan difundido incluso desde los organismos multilaterales ${ }^{(6)}$ indica: Abogar por la equidad en salud no puede consistir simplemente en demandas relacionadas con la distribución de la atención sanitaria en particular. Los factores que pueden contribuir a los logros y fracasos en el campo de la salud van mucho más allá de la atención sanitaria e incluyen muchas influencias muy distintas, desde las predisposiciones genéticas, los ingresos individuales, los hábitos alimentarios y los estilos de vida hasta el entorno epidemiológico y las condiciones de trabajo ${ }^{(5)}$.

Asi la equidad en salud incluye para Sen no solo el logro de la salud y la posibilidad concreta de todos para alcanzarla, entre otros factores con una adecuada distribución de los servicios, sino también la justicia del proceso, que elimine cualquier discriminación al tiempo que se integra en el concepto más amplio de justicia social, evitando cualquier tipo de reduccionismo que la identifique con uno $u$ otro de sus componentes. Las reflexiones de Sen son profundamente iluminadoras pero no dan soluciones específicas para alcanzar la equidad en salud, su esfuerzo muestra la complejidad y multidimensionalidad del proceso y por tanto la necesidad de su adaptación a realidades concretas.

Si bien el debate en latinoamerica sobre la equidad en salud no ha estado ausente ${ }^{(7)}$, para el caso de países como el Perú con una herencia colonial y una composición multiétnica, la equidad en salud debe contemplar las profundas diferencias generadas no solo por las disparidades económicas y sociales sino aquellas derivadas de la diversidad cultural, tema que no es ajeno a contextos del primer mundo ${ }^{(8)}$.

Es ampliamente conocido que las estadísticas acumulativas de la realidad sanitaria de los países con población indígena invisibilizan las enormes inequidades que separan a pueblos indígenas no solo con las poblaciones urbanas sino con poblaciones comparables por su ruralidad.

\section{EQUIDAD EN SALUD EN LOS 90}

La oficina regional de la OMS en Europa publicó en 1990 un documento técnico de Whitehead referido a la inequidad en salud que ha tenido innumerables citas, en particular en su definición de inequidad en salud: el término inequidad tiene una dimensión moral y ética. Se refiere a las diferencias que son innecesarias $y$ evitables, pero, que además, también se consideran injustas. Por lo tanto, con el fin de describir una determinada situación como injusta, la causa tiene que ser examinada y juzgada como injusta en el contexto de lo que está pasando en el resto de la sociedad ${ }^{(9)}$.

El documento correspondía a un análisis de la realidad europea pero, traducido a más de veinte idiomas, se difundió muy ampliamente en América y Asia donde su influencia ha sido notable. Su énfasis en el carácter ético y moral la ubica en la tradición filosófica occidental ya revisada pero su referencia a "diferencias evitables e innecesarias" buscaba una operatividad sobre la que continúa el debate. Si bien siempre se había reconocido que la salud de las personas pobres es menor que la de las clases más favorecidas, otras diferencias se referían a la dualidad urbano-rural, o a ámbitos regionales concretos en un mismo país y en general a diversas diferencias evidentes. Refiriéndose a lo que debe considerarse equidad en salud lo define en tres términos de igualdad: igualdad de acceso a la atención disponible para igual necesidad, igualdad de uso para igual necesidad e igual calidad de atención para todos

Esta propuesta ha recibido críticas por centrarse excesivamente en la prestación de servicios de salud, pero veinte años después un nuevo artículo de Whitehead y Dahlgren reactualiza su posición. En primer lugar operativiza de modo distinto la equidad en salud: el concepto de la equidad en salud implica que, en una situación ideal, todas las personas alcanzan un grado máximo de salud y ninguna se ve en situación de desventaja por razón de su posición social o por otras circunstancias determinadas por factores sociales (11). 
En segundo lugar zanja una disquisición terminológica: En anteriores documentos publicados por los autores se utilizó el término inequidades en salud (en inglés, inequities in health), aunque explicándose que en algunos países, sobre todo en el Reino Unido, se utilizaba también, y con el mismo significado, el término desigualdades en salud (en inglés, inequalities in health) ... En este documento, para mantener la coherencia con otros documentos de la Organización Mundial de la Salud, se mantiene el término inequities in health. No obstante, los autores desean insistir en que, en el ámbito de la salud pública, el término desigualdades sociales en salud tiene la misma connotación que el de diferencias de salud injustas ${ }^{(10)}$.

Estas precisiones se incorporan en el Glosario de Promoción de la salud de la OMS, en el cual se define la Equidad en salud diferenciándola del estado de salud: Equidad en salud no es lo mismo que igualdad en el estado de salud. Las desigualdades en cuanto al estado de salud entre los individuos y las poblaciones son consecuencias inevitables de las diferencias genéticas, de diferentes condiciones sociales y económicas o de elecciones de un estilo de vida personal. La falta de equidad tiene lugar como consecuencia de las diferencias de oportunidades derivadas, por ejemplo, del acceso desigual a los servicios de salud, a una alimentación correcta, a una vivienda adecuada, etc. En tales casos, las desigualdades en cuanto al estado de salud surgen como consecuencia de la falta de equidad en materia de oportunidades en la vida ${ }^{(11)}$.

Hay un largo camino recorrido desde la discusión estrictamente filosófica sobre la justicia y su aplicación superior: la equidad, hasta las actuales discusiones operativas de cómo conseguir la equidad en salud. Es claro que la inequidad en salud es multidimensional y que a su vez la equidad en salud es una condición necesaria para la justicia social, el reto en países complejos cultural y étnicamente que además parten de una situación de enormes disparidades económicas y sociales es encontrar formas concretas y específicas, consensuadas sobre lo que coincidimos en identificar como inequidades en salud, la construcción de una salud equitativa pasa por ese consenso previo producto a su vez de una reflexión colectiva que abarque nuestras diversidades y no aplique mecánicamente criterios economicistas o etnocéntricos. La reforma en salud que se desarrolla en diversos países latinoamericanos es una oportunidad que no debemos perder para avanzar hacia la equidad en salud.

\section{REFERENCIAS BIBLIOGRÁFICAS}

1. Sófocles. Antígona. Madrid : Ed. Gredos; 2010

2. Fórmula para el interrogatorio ritual de los enfermos. En: Clásicos de la Medicina. Nínive, Grecia: Biblioteca de Asurbanipal; Siglo VII a. C.

3. Aristóteles. Ética Nicomaquea. Libro V. En: Aristoteles. Obras. Madrid: Ed. Aguilar Trad P. Samaranch; 1973.

4. Rawls J. A Theory of Justice (revised edition). Oxford: Oxford University Press; 1999.

5. Sen A. ¿Por qué la equidad en salud? Rev Panam Salud Publica. 2002;11(56):302-9.

6. Drane JF. El desafío de la equidad: una perspectiva. En: Lolas F (ed). Bioética y cuidados de la salud. Equidad, calidad y derechos. Washington: OPS; 2000. p. 75-89.

7. Hernandez-Alvarez M. El concepto de equidad y el debate sobre lo justo en salud. Rev Salud Pública. 2008;10 Sup 1:72-82.

8. Hebert PL, Sisk JE, Howell EA. When does a difference become a disparity? Conceptualizing racial and ethnic disparaties in health. Health Aff (Millwood). 2008;27(2):374-82. doi: 10.1377/hlthaff.27.2.374.

9. Whitehead M. The concepts and principles of equity and health. Copenhagen: World Health Organization Regional Office for Europe; 2000
10. Whitehead M. Dahlgren G. Conceptos y principios de la lucha contra las desigualdades sociales en salud: Desarrollando el máximo potencial de salud para toda la población. Madrid: Ministerio de Sanidad y Política Social; 2010.

11. Organización Mundial de la Salud. Promoción de la salud. Glosario. Ginebra: OMS; 1998.

Correspondencia: Oswaldo Salaverry García

Dirección: Calle Cápac Yupanqui 1400, Lima 11, Perú.

Teléfono: (511) 748-0000 Axo: 1345

Correo electrónico: oswaldosalaverry@ gmail.com 